monoculture (IL-1 $\beta$ (75\%), activin A $(22 \%)$, activin A and IL$1 \beta(101 \%)$, follistatin and IL-1 $\beta(67 \%))$.

Conclusions The autoregulatory cycle of activin A and follistatin is active in HUVECs, but not in RASF. In direct coculture of HUVECs and RASF, the effects of HUVECs appear to overweigh resulting in a significant reduction of IL- $1 \beta$ in the presence of follistatin.

Disclosure of Interest None declared.

\section{P081/020 LASP1 REGULATES CELL-TO-CELL CONTACT FORMATIONS OF FIBROBLAST-LIKE SYNOVIOCYTES IN RA}

${ }^{1} \mathrm{D}$ Beckmann*, ${ }^{1} \mathrm{~A}$ Krause, ${ }^{1} \mathrm{U}$ Hansen, ${ }^{2} \mathrm{HP}$ Kiener, ${ }^{3} \mathrm{~T}$ Kamradt, ${ }^{1} \mathrm{~T}$ Pap, ${ }^{1} \mathrm{~A}$ Korb-Pap. ${ }^{1}$ Institue of Musculoskeletal Medicine, University Hospital Muenster, Muenster; ${ }^{2}$ Department of Medicine III, Division of Rheumatology, Medical University of Vienna, Vienna; 3 Institute of Immunology, University Hospital of Jena, Jena, Germany

10.1136/annrheumdis-2018-EWRR2019.70

Career situation of first and presenting author Post-doctoral fellow.

Introduction In rheumatoid arthritis (RA), fibroblast-like synoviocytes (FLS) undergo a stable transformation resulting in an aggressive, tumour-like phenotype that mediates cartilage damage by increased levels of MMPs and adhesion molecules such as $\beta 1$ integrins. In this context, the tumour-associated protein Lasp1 is of interest because it modulates actin organization and focal adhesion turnover.

Objectives The effects of Lasp1 deficiency on RA-FLS cell-tocell contact formations, the disease course and joint destruction have been investigated in this study.

Methods Lasp-1 expression was analysed in RA synovial tissue and in murine models of arthritis (hTNFtg mice and G6PI mouse model). Hind paws were analysed by using WB analyses and immunofluorescence stainings, primary FLS were isolated and cultivated, respectively. Furthermore, Lasp $1^{-/-}$mice were interbred with hTNFtg mice and offsprings were analysed for the progression of joint destruction by clinical evaluation and histopathology. Migration characteristics of FLS derived from wild type (wt), Lasp $1^{-/}$, hTNFtg and Lasp $1^{-/}$hTNFtg mice were analysed by live cell imaging. Additionally, we used an in vitro 3D organ culture system for functional analyses.

Results Upregulated Lasp1 levels in RA synovial tissue and FLS were observed. In line with the human data, increased levels of Lasp1 were found in murine FLS derived from hTNFtg mice and the chronic G6PI model. Lasp1 was located in structures of cell-matrix as well as cell-to-cell contacts. The loss of Lasp1 led to clear alterations in adherens junction arrangement indicated by altered $\beta$-catenin pattern. In vivo evaluation of $\mathrm{Lasp} 1^{-/}$hTNFtg mice revealed a milder arthritis score, less cartilage degradation and reduced FLS attachment to articular cartilage compared to hTNFtg mice. In vitro migration assays using live cell imaging demonstrated alterations in spreading morphology and cell-to-cell contact turnovers and a significantly reduced migration rate of Lasp1 $1^{-/}$ FLS and Lasp $1^{-/}$hTNFtg FLS compared to controls $(-69.11 \%$ after 24 hour). Histological sections of the 3D matrices demonstrated that wt FLS formed an organised synovial structure comparable with healthy synovial tissue in vivo, whereas in matrices with hTNFtg FLS this synovial architecture absent. Interestingly, Lasp1 deletion in the hTNFtg background resulted in an organised cellular lining layer comparable with wt FLS matrices.
Conclusions Lasp1 represents an interesting target involved in RA-caused joint destruction, because its loss resulted in significantly reduced cartilage destruction in vivo and RA-FLS interactions and migration rates in vitro.

Disclosure of Interest None declared.

\section{P082/024 SCLEROSTIN DEFICIENCY AFFECTS RANKL-MEDIATED OSTEOCLAST DIFFERENTIATION}

IJ Intemann*, ${ }^{1} \mathrm{C}$ Wehmeyer, ${ }^{1} \mathrm{DJ}$ de Gorter, ${ }^{1} \mathrm{~V}$ Kracke, ${ }^{1} \mathrm{P}$ Paruzel, ${ }^{2} \mathrm{I}$ Kramer, ${ }^{2} \mathrm{M}$ Kneissel, ${ }^{1} \mathrm{~T}$ Pap, ${ }^{1} \mathrm{~B}$ Dankbar. 'Institute of Musculoskeletal Medicine, University Hospital Muenster, Muenster, Germany; ${ }^{2}$ Novartis Institutes for BioMedical Research, Basel, Switzerland

\subsection{6/annrheumdis-2018-EWRR2019.71}

Career situation of first and presenting author Student for a master or a $\mathrm{PhD}$.

Introduction Sclerostin is a Wnt inhibitor and has anti-anabolic effects on bone formation by negatively regulating osteoblast differentiation. Sclerostin loss-of-function leads to a higher bone mass and bone strength. Therefore, inhibition of sclerostin is currently considered as a promising treatment for osteoporosis. Surprisingly, in a TNF $\alpha$-dependent arthritis mouse model (hTNFtg) the genetic deficiency of sclerostin caused a deterioration of disease severity. hTNFtg mice lacking sclerostin displayed enhanced and bone erosion associated with an elevated number of osteoclasts within the joint.

Objectives In order to understand the underlying mechanisms, we aimed to investigate the direct and indirect impact of sclerostin on osteoclast differentiation and bone erosion in arthritis. Methods Sclerostin knockout $\left(\right.$ sost $^{-/}$) mice were crossbred with hTNFtg mice to obtain sost $t^{-/} / \mathrm{hTNF}$ tg mice, from which synovial fibroblasts (SF) were isolated. Cocultures of synovial fibroblasts and green fluorescent protein $\left(\mathrm{GFP}^{+}\right)$bone marrowderived macrophages (BMM) were performed and osteoclastogenesis was analysed. Receptor activator of NF-kB ligand (RANKL) and macrophage colony stimulating factor (MCSF) expression was measured by ELISA and IL-1a expression by Western Blot. Viability of osteoclasts precursors was measured by MTT Assay.

Results In cocultures of SF and $\mathrm{GFP}^{+} \mathrm{BMM}$, osteoclast formation was enhanced by $s o s t^{-/} / \mathrm{hTNF}$ tg SF compared to hTNFtg SF. Expression of RANKL and MCSF, two crucial factors for osteoclast differentiation, was not different between the genotypes. Interestingly, stimulation of wildtype BMM with conditioned media (CM) from hTNFtg or sost $^{-/ /} / \mathrm{hTNF}$ tg synovial fibroblasts showed no $\mathrm{TRAP}^{+}$cells at all. However, CM supplemented with RANKL lead to an elevated number of osteoclasts using sost $t^{-/} / \mathrm{hTNFtg} \mathrm{CM}$ compared to hTNFtg CM, pointing to a secreted factor promoting osteoclast development. In this regard, the osteogenic factor IL- $1 \alpha$, was higher expressed in sost ${ }^{-/} / \mathrm{hTNFtg}$ SF than in hTNFtg SF. Moreover, the treatment of cocultures with recombinant sclerostin lead to a decreased number of osteoclasts in both genotypes. Accordingly, sclerostin inhibited osteoclastogenesis in monocultures when administered in the pre-differentiation phase, whereas no effect was observed in the differentiation phase, indicating an inhibitory effect of sclerostin mainly on osteoclast precursors.

Conclusions Sclerostin deficiency in hTNFtg SF promotes RANKL-mediated osteoclast differentiation, which is likely dependent on the inhibitory effect of sclerostin itself and/or on the promoting effect of higher levels of IL- $1 \alpha$.

Disclosure of Interest None declared. 